\title{
Multislice Spiral Computed Tomography under Hybrid Iterative Reconstruction Algorithm in Staging Diagnosis of Bladder Cancer and Ceramide Glycosylation
}

\author{
Jun Liu $\mathbb{D}^{1}$ and Xiaolong Jiang $\mathbb{D}^{2}$ \\ ${ }^{1}$ Department of Urology, The Second Affiliated Hospital, Hengyang Medical School, University of South China, Hengyang 421001, \\ Hunan, China \\ ${ }^{2}$ Department of Radiology, Affiliated Nanhua Hospital, University of South China, Hengyang 421002, Hunan, China
}

Correspondence should be addressed to Xiaolong Jiang; 2011020016@usc.edu.cn

Received 7 July 2021; Revised 3 September 2021; Accepted 6 September 2021; Published 21 September 2021

Academic Editor: Gustavo Ramirez

Copyright ( $\odot 2021$ Jun Liu and Xiaolong Jiang. This is an open access article distributed under the Creative Commons Attribution License, which permits unrestricted use, distribution, and reproduction in any medium, provided the original work is properly cited.

\begin{abstract}
This study was to discuss the application of multislice spiral computed tomography (CT) in the staging diagnosis of bladder cancer and the effect of ceramide glycosylation. The hybrid iterative reconstruction algorithm was applied. Immunohistochemistry and western blot were used to detect the normal bladder tissues (30 cases) of GCS in group 1 (100 cases) and group 2. The scanned images of all the research objects were obtained, the images with the iterative reconstruction algorithm were reconstructed, and statistical analysis on the CT value under the algorithm was conducted. The results showed that the image quality, blood vessel sharpness, average image score, signal-to-noise ratio, and radiation dose after the spiral CT and iterative reconstruction algorithm all increased, while the noise value decreased. The optical density value of glucosylceramide synthase in group 2 patients increased by $71 \%$, and the optical density value of group 1 increased by $29 \%$. The optical density expression of glucosylceramide synthase in group 1 patients was significantly higher than that in the control group, and there was a statistical difference between the two $(P<0.05)$. Among the results of multislice spiral CT for tumor staging, the lesions larger than $5 \mathrm{~cm}$ and in the range of $1.1-2 \mathrm{~cm}$ in diameter were more sensitive. In 41 patients, there were multiple lesions. A total of 142 cancer lesions were found. The diameter of the tissue ranged from 0.5 to $6.8 \mathrm{~cm}$, with an average diameter of $2.03 \pm 0.35 \mathrm{~cm}$. The optical density of glucosylceramide synthase in the group 1 was 5526, and the optical density in group 2 was 2576. The OD expression of GCS in group 1 was greatly higher in contrast to that in group 2, and there was a statistical difference between the two groups $(P<0.05)$. The multislice spiral CT examination under this algorithm found that the diagnosis and staging accuracy of lesions with a diameter greater than $5 \mathrm{~cm}$ and tumor diameters in the range of 1.1 to $2 \mathrm{~cm}$ was higher. The image processed by the hybrid iterative reconstruction algorithm had good effect, high definition, and accuracy.
\end{abstract}

\section{Introduction}

Bladder cancer is a malignant tumor that occurs on the bladder mucosa. It is the most common malignant tumor in the urinary system and one of the ten most common tumors throughout the body. The etiology of bladder cancer is extremely complex, which is closely related to both internal genetic factors and external environmental factors [1]. The two basic and relatively clear risk factors for bladder cancer are smoking and occupational exposure to aromatic amine chemicals [2]. The initial clinical manifestations of patients with bladder cancer are hematuria, which is usually painless, intermittent, and visible hematuria and can be relieved or stopped by itself [3]. There may also be symptoms of bladder irritation (frequent urination, urgency, painful urination, etc.), dysuria, upper urinary tract obstruction, lower abdominal masses, and so on. In severe cases, tumor metastasis may occur [4]. Clinical diagnosis of bladder cancer and its staging requires comprehensive consideration of the patient's past and family history, combined with symptoms 
and physical examination to make a preliminary judgment, and further related examinations [5]. The examination methods include routine urine examination, urine exfoliative cytology, urine tumor markers, and abdominal and pelvic ultrasound examinations [6]. Based on the results of the above examinations, it is determined whether to perform cystoscopy, intravenous urography, pelvic computed tomography (CT), or pelvic magnetic resonance imaging (MRI) to confirm the diagnosis [7]. The clinical treatment of bladder cancer relies on a variety of antitumor and antiinfective drugs, which greatly increases the probability of patients developing drug-resistant bacteria. Studies have found that ceramide glycosylation can lead to multidrug resistance in tumors; ceramide is a lipid molecule produced by the hydrolysis of cell membrane sphingomyelin, and it can act as a second messenger in the process of apoptosis and promote tumor cell proliferation[8]. Studies have shown that the increased activity of glucosylceramide synthase (GCS) in multidrug-resistant cells will convert ceramide to glucosylceramide, which can cause the failure of anticancer drugs [9]. If the ceramide glycosylation process of this cell can be inhibited, the multidrug resistance of tumor cells can be reversed [10].

With the rapid development of electronics technology and computer technology, the basic theories and settings of CT research have been improved. As early as the 1990s, a multislice spiral CT appeared. It has a multirow wide detector structure, a tube that can be exposed at one time, and an imaging system that can obtain multiple layers of image data synchronously. Cystoscopy may cause some damage or injury, but the multislice spiral CT is more practical and exerts the prompting effect on the tissue infiltration around bladder cancer $[11,12]$. However, the imaging principle of CT scanners is the strong penetrating ability of X-rays, so the ionizing radiation to the patient is increased during the examination, which inevitably causes harm to the patient. Therefore, maintaining high definition image with reduced scanning dose has become a problem that needs to be solved urgently [13]. Hybrid iterative reconstruction algorithm is an alternative image reconstruction method, which allows imaging under a lower radiation dose with similar noise level and image quality, so it is beneficial to reduce fringe artifacts and speckle noise in the image to correct or prevent deviation artifacts, showing high practical value in improving CT scan results [14].

The iterative reconstruction algorithm is a new generation of IR technology, which can obtain images that meet the diagnostic requirements under lower scanning conditions. Although the iterative algorithm has a large amount of calculation and takes a long time, it is still considered to be a better reconstruction method for incomplete projection and noisy projection data. For the optimization of the CT reconstruction algorithm, it uses the original CT collected data; through repeated mathematics and physics, it can greatly reduce the noise value on the image, improve the signal-tonoise ratio, and reduce the radiation dose received by the patient. The image quality is optimized, and the spatial resolution and density resolution of the image are improved. Therefore, it is of great significance to study efficient CT reconstruction algorithms, make full use of the advantages of the algorithms, and apply them flexibly to the clinic.

There are many diagnostic methods for bladder cancer at home and abroad, such as free DNA and DW-MRI, but the application of hybrid iterative algorithms in the diagnosis of bladder cancer is relatively small. Therefore, the multislice spiral CT with hybrid iterative reconstruction algorithm was adopted in this study for staging diagnosis of bladder cancer and to explore the effects of ceramide glycosylation, aiming to provide clinical reference for the diagnosis and treatment of bladder cancer.

\section{Information and Methods}

2.1. Basic Information. 100 patients with bladder cancer admitted to the hospital from January 2018 to January 2020 were selected and included in the experimental group (group 1 ), and 30 normal people who participated in the physical examination at the hospital during the same period were selected and included in the control group (group 2). There were 63 males and 37 females in group 1, aged 31-82 years, with an average age of $53.27 \pm 3.94$ years. The clinical manifestations of them were hematuria, including 23 patients with dysuria, 10 patients with lower abdominal pain, and 36 patients with fever. All patients were pathologically confirmed by cystoscopy biopsy. In group 2, there were 14 males and 16 females, aged 33-79 years, with an average of $50.14 \pm 3.07$ years. There was no statistically obvious difference in basic information between the two groups of patients $(P>0.05)$, and the two groups were comparable (Table 1). The medical ethics committee of our hospital reviewed and approved this study. The diagnosis was performed by referring to the relevant diagnostic criteria in the Guidelines for the Diagnosis and Treatment of Bladder Cancer. The inclusion criteria were defined as follows: those who met the above diagnostic criteria; those who were diagnosed for the first time; those who had indications for surgery; and those who were aware of and signed the informed consents. The exclusion criteria were determined as follows: those with underlying diseases of other important organs; patients with poor compliance and who were unable to cooperate with the investigator; those with cognitive impairment; and those with congenital heart disease, etc.

2.2. Scanning Method of Enhanced Multislice Spiral CT. The patient had to make necessary preparation before the examination according to the doctor's advice. They had to keep fasting for 8 hours and then drink $1000 \mathrm{~mL}$ of water 2 hours before the examination to keep the bladder in a full state. At the beginning of the scan, the bladder was scanned using a multislice spiral CT scanner (manufacturer: BRUKER (Beijing in China); model: Optima CT660) from the lower edge of the ulnar symphysis to the level of the xiphoid process. The scanning parameters were set as follows: tube current was $150 \mathrm{~m}$. As the tube voltage was $100 \mathrm{kV}$, the layer thickness was $5.0 \mathrm{~mm}$, the layer spacing was 2.5-5.0 $\mathrm{mm}$, the thread pitch was $1 \mathrm{~mm}$, and the matrix was $512 \times 512$. When the ray was converted into visible light, the 
TABle 1: Comparison of general information.

\begin{tabular}{lcccccc}
\hline & Age $(x \pm s)$ & Male & Female & Lower abdominal pain & Patients with fever & Difficulty urinating \\
\hline Control group & $50.14 \pm 3.07$ & 14 & 16 & & & 36 \\
Observation group & $53.27 \pm 3.94$ & 63 & 37 & 10 & 24 \\
$P$ & 0.623 & & & & \\
\hline
\end{tabular}

photoelectric sensor can be converted into an electrical signal, and then the information was converted into a digital signal by a digital-to-analog converter. Finally, the calculation reconstruction was completed by a simple computer equipped for the CT.

\subsection{Hybrid Iterative Reconstruction Algorithm Models.} The hybrid iterative reconstruction algorithm can better denoise the image, improve the image quality, and reduce radiation. The working principle of the hybrid iterative algorithm is to reduce the noise in the front and rear projection domain. At the beginning of each calculation, an image assumption had to be made that was to set a similar initial value for each image, and then the possible projection value after the ray passing through the human body was calculated. At the same time, the calculated result and the real projection result were analyzed to obtain the corresponding correction result, which could correct the pixel value multiple times until all the image reconstruction was completed. The specific principle of hybrid iteration is shown in Figure 1, and the specific equation of hybrid iteration was given in Figure 2. Figure 2 shows the schematic diagram of the equation. The first is the noise positive line graph, and then the iterative denoising graph is used to reduce the noise, and finally the reconstructed image is obtained.

It was found that the projector data received by the CT detector was very similar to the Gaussian distribution, and the mean and variance of the projection data after logarithmic transformation conformed to the following equation:

$$
R_{i}^{2}=A_{i} \times \exp \frac{B_{i}}{\eta}
$$

In equation (1), $R_{i}$ represented the mean value of the data received by the $i^{\text {th }}$ detector unit, and $R_{i}^{2}$ referred to the variance of the data. In the traditional algorithm, the weighted least-squares lattice (WLSL) can be used to reduce the image noise in the front and rear projection space.

$$
\begin{aligned}
& \phi(a)=(\hat{y}-a)^{\prime} \Sigma^{-1}(\hat{y}-a), \\
& \phi(b)=(\hat{y}-t b)^{\prime} \Sigma^{-1}(\hat{y}-t b) .
\end{aligned}
$$

Equation (2) was the iterative equation for the front projection domain (as shown in Figure 3), and equation (3) was the iterative equation for the back-projection domain (Figure 4). $A=t b$ referred to the vector of the complete projection data set, and it was estimated from the initial image; $v$ represented the final attenuation coefficient to be calculated; $t$ represented the projection matrix; and $y$ was the logarithmic result of the detector measurement data.
The penalty weighted least-squares method was added to the iterative algorithm of CT because the slightly smooth penalty factor can effectively improve the chessboard effect of the traditional least-squares method and ensure the performance of convergence:

$$
\phi(a)=(\hat{G}-a)^{\prime} \Sigma^{-1}(\hat{G}-a)+\beta K(a) .
$$

In this equation, $W$ represented the ideal value of the estimated projection data; $\Sigma^{-1}$ represented the noise variance matrix; $G$ was the projection data measured by the reagent, and the last term $K(a)$ referred to the marked penalty factor, which can control the convergence performance. In addition, $\beta$ can adjust the degree of calculation.

The iterative equation was given as follows:

$$
W_{l}^{(n+1)}=\frac{W_{l}+\beta \partial_{l}^{2}\left(\sum_{k \in J_{l}^{2}} A_{l j} Y_{j}^{n+1}+\sum_{k \in J_{l}^{2}} A_{l j} Y_{j}^{n}\right)}{1+\beta \partial_{l}^{2} \sum_{k \in J_{l}^{2}} A_{l j}} .
$$

In equation (5), $l$ represented the guide value of each pixel on the image; $J_{l}$ and $Y_{l}$ referred to the pixel value at the center of the image and the value of adjacent pixel, respectively; $A_{l j}$ represented the weight; $k$ represented the iteration period of the equation; and $A$ and $Y$ were left and right adjacent areas around the target pixel, respectively. The relationship between the preprocessed projection data and the estimated projection data could be expressed as follows:

$$
\begin{aligned}
T(A) & =\frac{1}{2} \sum \sum_{\substack{N u J_{l} \\
i}} A_{l j}\left(a_{l}-Y_{j}\right), \\
a & =\arg \min _{p \geq 0} \varphi(a) .
\end{aligned}
$$

The hybrid iterative reconstruction algorithm was compared with the filtered back-projection reconstruction algorithm in this study by measuring the noise of the reconstructed image and analyzing the performance with accuracy and sensitivity as indicators. The specific equations were as follows:

$$
\begin{aligned}
& A=\frac{M_{T}}{P} \times 100 \%, \\
& Q=\frac{P}{W+F} \times 100 \%, \\
& Y=\frac{N}{H+F} \times 100 \% .
\end{aligned}
$$

In the above equations, $A, Q$, and $Y$ referred to the accuracy, sensitivity, and specificity, respectively; $M_{T}$ meant the number of cases with accurate prediction, $T$ meant the total number of patients, and $P$ and $N$ represented the number of patients with true positive and true negative test result, 


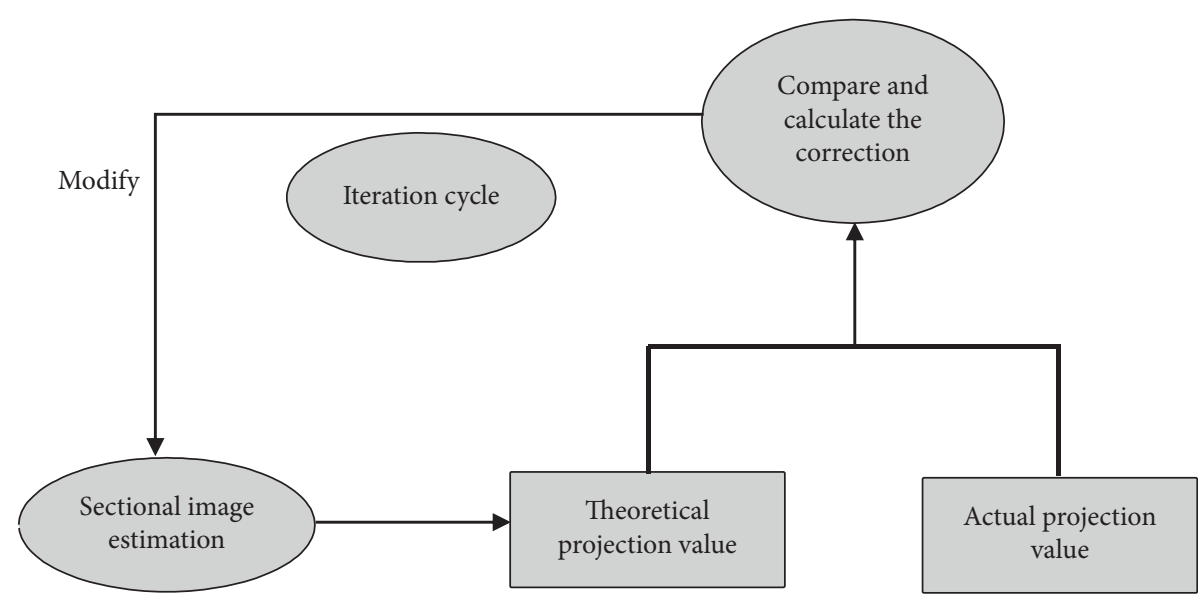

FIgURE 1: Schematic diagram of hybrid iteration principle.

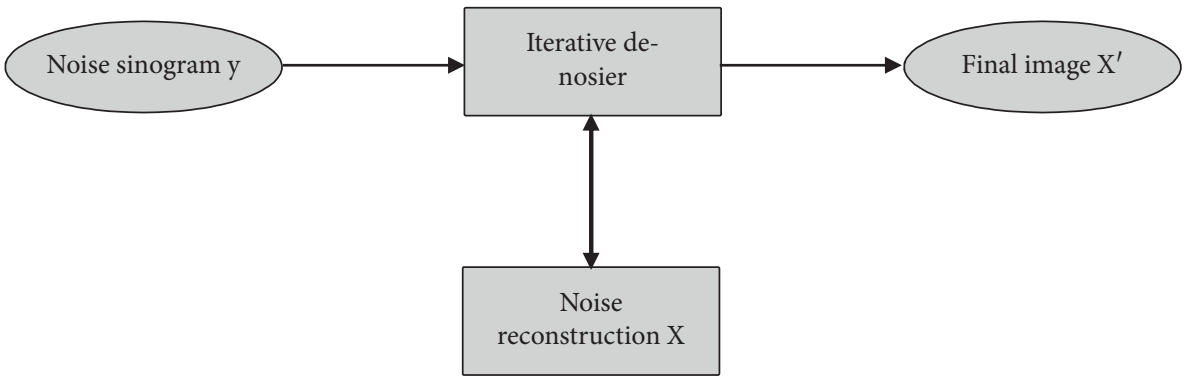

FIgURE 2: Schematic diagram of hybrid iteration equation.

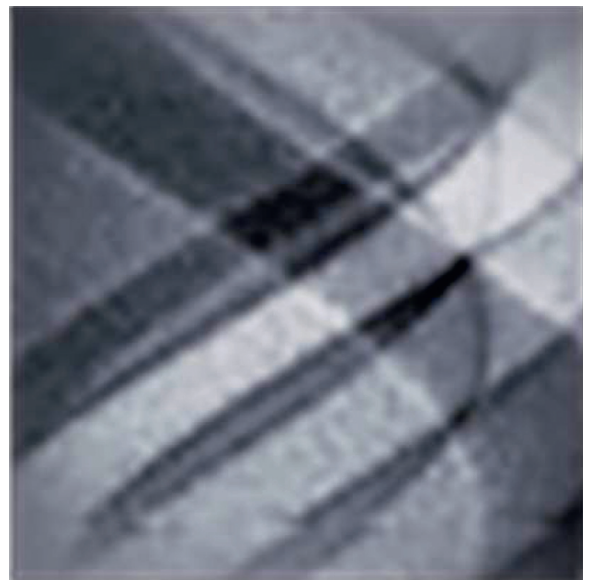

FIGURE 3: Iteration of front projection domain.

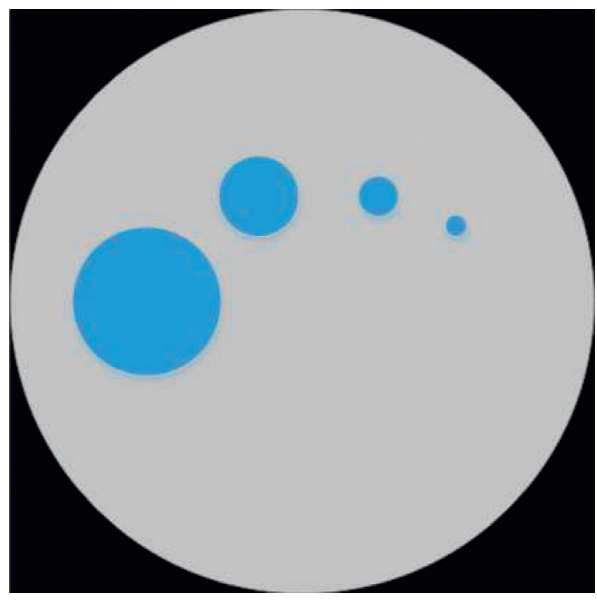

FIGURE 4: Iteration of rear projection domain. respectively; and $F$ and $H$ referred to the number of patients with false positive and false negative results, respectively.

For the study of ceramide glycosylation in patients with bladder cancer, transgenic technology was used to detect the expression level of glucosylceramide and the corresponding indicators after treatment with chemotherapy drugs.

2.4. Detection of Ceramide Glycosylation. The immunohistochemistry and western blot technology were applied to detect the expression of GCS in bladder tissues and normal tissues. Firstly, the bladder tissues of the two groups of objects were collected and prepared into specimens, which were stored in the refrigerator, embedded in paraffin, and baked in an oven at $60^{\circ} \mathrm{C}$ for 24 hours. The paraffin sections were routinely dehydrated. After washing for many times, they were kept at room temperature overnight. After treatment with DAB for color development for about 10 minutes, the sections were counterstained with hematoxylin, dried, and mounted to observe under a microscope. 
2.5. Staging of Bladder Cancer. The staging of bladder cancer mainly refers to the degree of invasion and metastasis. Bladder cancer is divided into T1-T4 stages. For Tx stage, the primary tumor cannot be evaluated; mainly T0 stage has no evidence of primary tumor; Ta stage refers to noninvasive papillary carcinoma; Ts is carcinoma in situ. T1 tumors mainly invade the subcutaneous connective tissue; T2 tumors invade the muscle layer and are divided into T2a and T2b stages. T2a tumors mainly invade the superficial muscle layer, and T2b mainly invade the deep muscle layer; T3 tumors mainly invade the tissues around the bladder; T4 stage invades the prostate, seminal vesicles, uterus, vaginal pelvic wall, and abdominal wall. $\mathrm{N}$ indicates whether the lymph node has metastasis. $M$ mainly indicates whether there is distant metastasis.

2.6. Statistical Methods. The SPSS 21.0 statistical software was adopted for data analysis, the measurement data was represented by $(\bar{x} \pm s)$ and compared using the $t$-test; and the count data was represented by [cases (\%)] and compared using $\chi^{2}$. The measurement data meeting the normal distribution was analyzed by analysis of variance, while the measurement data not meeting the normal distribution was analyzed by the rank sum test; and the count data was analyzed by the chi-square test. $P<0.05$ indicated that the difference was statistically significant.

\section{Results}

3.1. Pathological Examination Results of Patients in Group 1. The pathological examination (Figure 5) suggested that, among the 100 included patients, 59 patients had single lesions and 41 patients had multiple lesions. A total of 142 cancer lesions were found, and the average diameter of bladder cancer tissue was $2.03 \pm 0.35 \mathrm{~cm}$.

\subsection{Performance Analysis of Hybrid Iterative Reconstruction} Algorithm. As illustrated in Figure 6, the image quality, blood vessel sharpness, average image score, SNR, and radiation dose after using the algorithm were all increased, while the value of noise was reduced; in addition, the differences in noise, SNR, and radiation dose were statistically significant in contrast to those before application of the algorithm $(P<0.05)$.

\subsection{Multislice Spiral CT Scan Results Based on Hybrid Iterative} Reconstruction Algorithm. After using the reconstruction algorithm, 4 groups of CT images were randomly selected for analysis, as shown in Figure 7, the orange box indicated the location of the lesion, and the image was clearer after reconstruction. Figure 7 shows that the cauliflower-like mass on the right posterior wall of the bladder was about $1 \mathrm{~cm}$ in size without bleeding from the ulcer, and it was about $2 \mathrm{~cm}$ from the ureteral orifice. The anterior urethra was slightly narrowed, and the bladder was mildly congested. The pathological diagnosis was papillary hyperplasia of the urothelium, increased levels of epithelial cells, disordered arrangement, deep stained nuclei, and irregular mitoses.

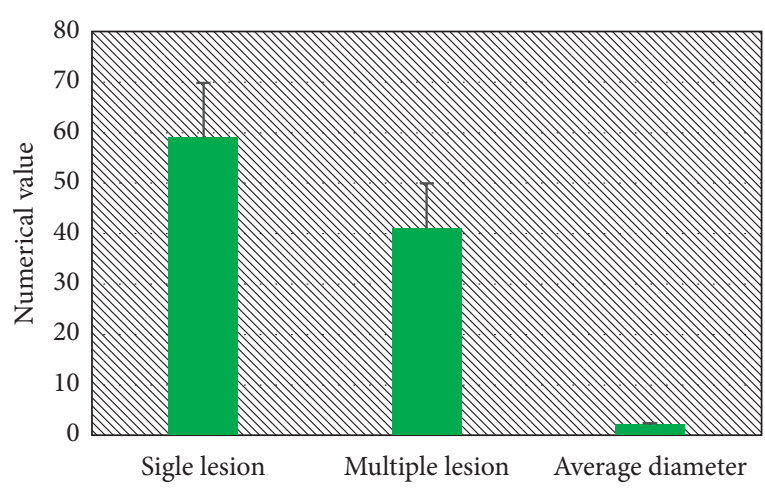

Figure 5: Pathological examination results of patients.

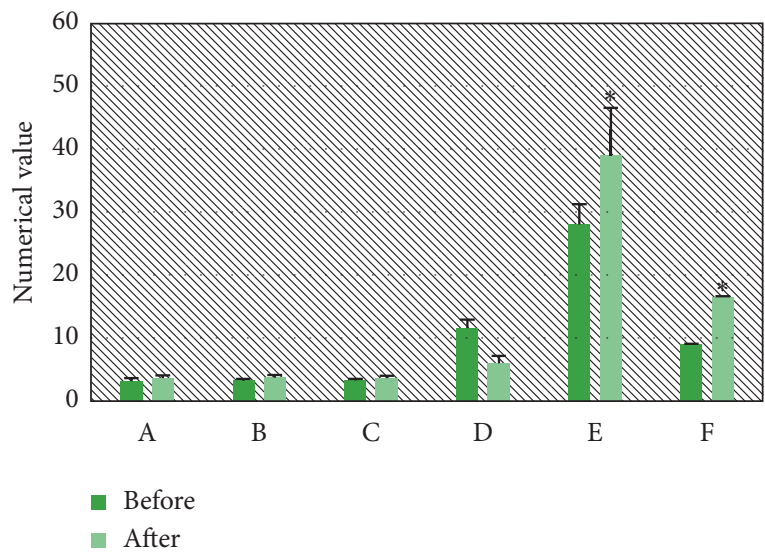

Figure 6: Performance analysis of hybrid iterative reconstruction algorithm. A B, C D, E, and F in the figure refers to image quality, blood vessel sharpness, average image score, noise, SNR, and radiation dose, respectively (* indicates that the difference is statistically significant, $P<0.05)$.

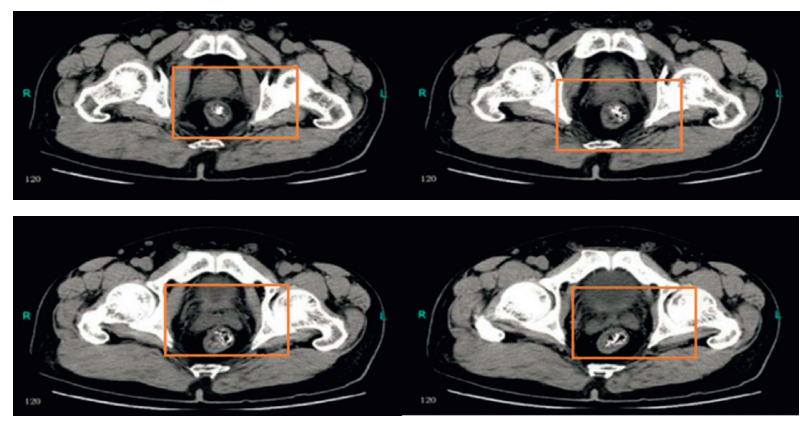

FIgURE 7: CT manifestations of bladder cancer.

3.4. Iterative Clinical Images. In the case of using the same dose in Figure 8, it showed that the image of the FBP algorithm had relatively large noise and strong graininess. In Figure 8(a), it used an iterative reconstruction algorithm, which greatly improved the image noise level and the smoothness of the image.

3.5. Application of Multislice Spiral CT Scanning in Tumor Staging Based on Hybrid Iterative Reconstruction Algorithm. As illustrated in Figure 9, the diameter range of more than 140 tumor lesions was $0.5-6.8 \mathrm{~cm}$. In the results of multislice 


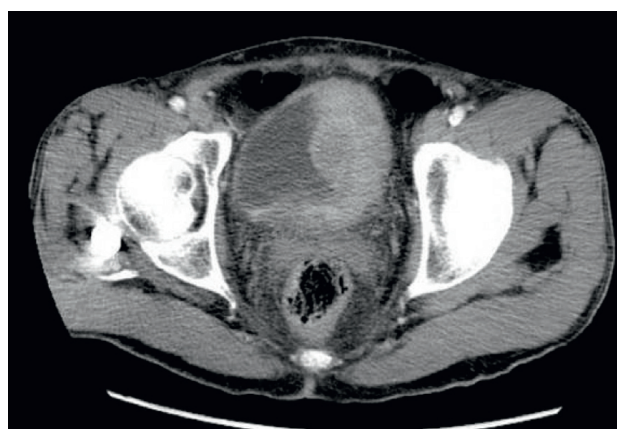

(a)

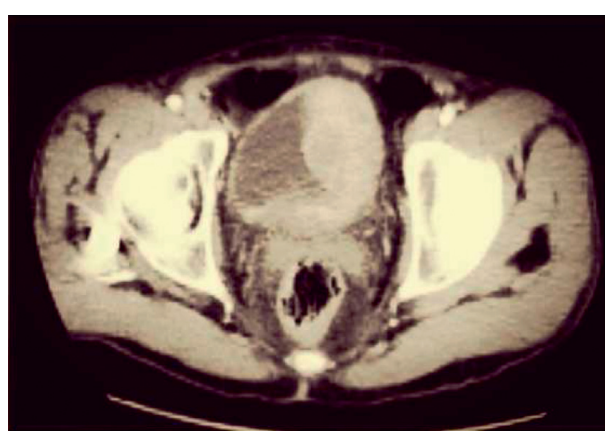

(b)

FIGURE 8: Iterative clinical images.

spiral CT for tumor staging, the more sensitive was the lesions larger than $5 \mathrm{~cm}$ in diameter, followed by the lesion with $1.1-2 \mathrm{~cm}$, and the difference was statistically obvious $(P<0.05)$.

3.6. Expression of GCS. Figure 10 shows the expression level of GCS in bladder tissues for subjects in group 1 and group 2, which was detected using the immunohistochemistry. It disclosed that GCS showed a higher expression in bladder cancer tissues, but its expression level was lower in normal bladder tissues.

3.7. Comparison on OD Results of GCS. As given in Figure 11, the optical density of glucosylceramide synthase in group 1 was 5526, and the optical density in group 2 was 2576 . The OD expression of GCS in group 1 patients was greatly higher in contrast to that in group 2, and there was a statistical difference between the two groups $(P<0.05)$.

\section{Discussion}

Bladder cancer is a very common malignant tumor in the clinic. In recent years, the incidence of this disease has gradually increased, and it has gradually become the first tumor of the urinary system [15]. The first response to bladder cancer is to use clinical examination methods to determine the stage of the disease and make timely and effective prevention. A multislice spiral CT was adopted for examination in this study, and a hybrid iterative reconstruction algorithm was applied to improve the sharpness and SNR of the image while reducing the radiation dose. It was found that, compared with traditional algorithms, the accuracy, sensitivity, and specificity of this algorithm were improved [16]. Gardner et al. [17] used an iterative reconstruction algorithm to perform image quality on prostate cancer patients, and the results showed that the Dice of the iterative reconstruction algorithm was increased by about $2.4 \%$, and the image uniformity was improved. The noise level and the overall image were improved. Miller et al. [18] used the CT reconstruction algorithm to analyze the automatic segmentation performance of the image, and the iterative reconstruction algorithm had greatly improved the automatic segmentation

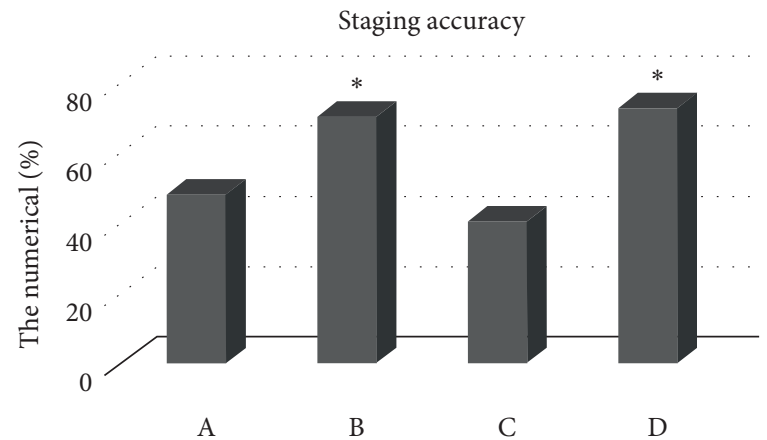

Figure 9: Tumor staging results. A, B, C, and D in the figure refers to the number of lesions with diameters of $0.5-1 \mathrm{~cm}, 1.1-2 \mathrm{~cm}$, $2.1-5 \mathrm{~cm}$, and $>5 \mathrm{~cm}$, respectively. ${ }^{*}$ suggests that $P<0.05$, which meant that the staging accuracy showed statistical difference in contrast to that of the lesion with another diameter.
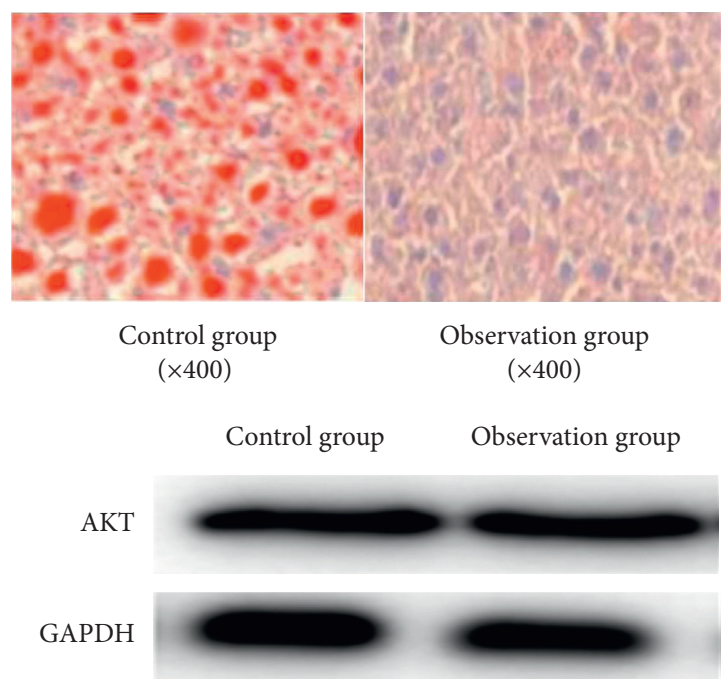

FIgURE 10: Expression of GCS.

performance. The results of this study also showed that the image quality, blood vessel sharpness, average image score, $\mathrm{SNR}$, and radiation dose of the algorithm were all increased, while the value of noise was reduced, suggesting that the algorithm showed obvious advantages in image 


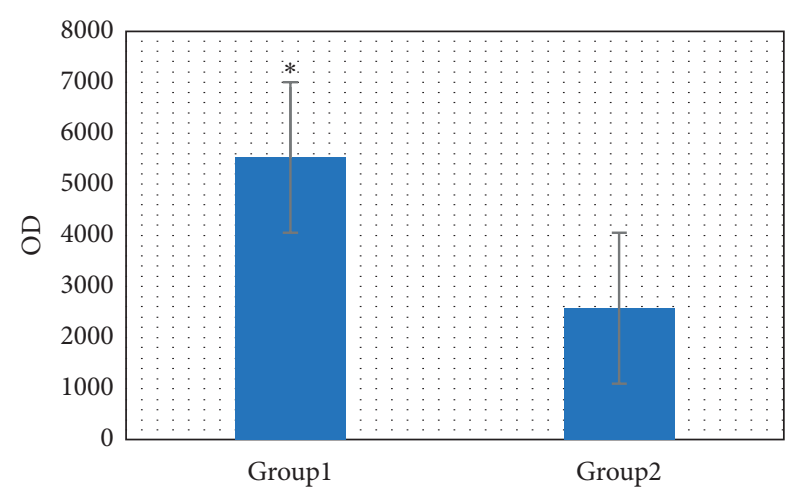

FIgURE 11: Comparison on OD results of GCS (* indicates that the difference is statistically significant, $P<0.05)$.

processing. The results of multislice spiral CT examination based on the hybrid iterative reconstruction algorithm revealed that the diameter of more than 140 tumor lesions ranged in $0.5-6.8 \mathrm{~cm}$. The results of multislice spiral CT for tumor staging disclosed that the lesions larger than $5 \mathrm{~cm}$ in diameter showed higher sensitivity, followed by the lesions with diameter of $1.1-2 \mathrm{~cm}$, showing statistically obvious differences in contrast to the staging accuracy on other size lesions $(P<0.05)$. Studies showed that the accuracy of CT detection for pedicled basal staging is higher, which may be related to the simple structure of pedicled basal tumor tissue.

In the treatment of bladder cancer, it is necessary to frequently check the blood routine and biochemical indicators of the patient [19]. Ceramide can induce cell apoptosis, and its synthetase can generate glucosylceramide by catalyzing sugar groups and combining with ceramide [20-22], while glucosylceramide can promote cell proliferation and reduce the therapeutic effect of anticancer drugs [23]. In this study, the immunohistochemistry was adopted to detect the expression of GCS in the bladder tissues of the patients in two groups. The results revealed that GCS showed a higher expression in bladder cancer tissues, but its expression level was lower in normal bladder tissues. In addition, the optical density (OD) of GCS in group 1 was much higher than that in group 2, showing statistically obvious difference. Such results suggested that ceramide synthase is closely related to the therapeutic effect of bladder cancer, which also provides a reliable reference value for clinical treatment.

\section{Summary}

In this research, based on the multislice spiral CT of the hybrid iterative reconstruction algorithm, it diagnosed the staging and retrograde of bladder cancer patients and then studied the effect of ceramide glycosylation on the treatment effect of the patients. It found that the diagnosis and staging rate of lesions with a diameter greater than $5 \mathrm{~cm}$ and tumor diameters in the range of 1.1 to $2 \mathrm{~cm}$ was higher; and the study of ceramide glycosylation indicated that ceramide synthase was closely related to the treatment of bladder cancer. The disadvantage of this study is that the sample size of the study is small, which leads to a certain deviation in the results of the study. Therefore, the sample size needs to be expanded for further study.

\section{Data Availability}

The data used to support the findings of this study are available from the corresponding author upon request.

\section{Conflicts of Interest}

The authors declare no conflicts of interest.

\section{References}

[1] V. G. Patel, W. K. Oh, and M. D. Galsky, "Treatment of muscle-invasive and advanced bladder cancer in 2020," CA: A Cancer Journal for Clinicians, vol. 70, no. 5, pp. 404-423, 2020.

[2] Y.-Y. Liu, R. A. Hill, and Y.-T. Li, "Ceramide glycosylation catalyzed by glucosylceramide synthase and cancer drug resistance," Advances in Cancer Research, vol. 117, pp. 59-89, 2013.

[3] J. P. Madigan, R. W. Robey, J. E. Poprawski et al., "A role for ceramide glycosylation in resistance to oxaliplatin in colorectal cancer," Experimental Cell Research, vol. 388, no. 2, Article ID 111860, 2020.

[4] V. Gupta, K. N. Bhinge, S. B. Hosain et al., "Ceramide glycosylation by glucosylceramide synthase selectively maintains the properties of breast cancer stem cells," Journal of Biological Chemistry, vol. 287, no. 44, pp. 37195-37205, 2012.

[5] H. Juri, Y. Narumi, V. Panebianco, and K. Osuga, "Staging of bladder cancer with multiparametric MRI," British Journal of Radiology, vol. 93, no. 1112, Article ID 20200116, 2020.

[6] Y.-Y. Liu, "Resuscitating wt expression by disrupting ceramide glycosylation: a novel approach to target mutant p53 tumors: figure 1," Cancer Research, vol. 71, no. 20, pp. 6295-6299, 2011.

[7] S. A. F. Morad, T. E. Ryan, P. D. Neufer et al., M. Kester, D. F. Claxton, B. M. Barth, T. G. Deering, and M. C. Cabot, Ceramide-tamoxifen regimen targets bioenergetic elements in acute myelogenous leukemia," Journal of Lipid Research, vol. 57, no. 7, pp. 1231-1242, 2016.

[8] V. Gupta, G. A. Patwardhan, Q.-J. Zhang, M. C. Cabot, S. M. Jazwinski, and Y.-Y. Liu, "Direct quantitative determination of ceramide glycosylation in vivo: a new approach to evaluate cellular enzyme activity of glucosylceramide synthase," Journal of Lipid Research, vol. 51, no. 4, pp. 866-874, 2010.

[9] J. Greffier, A. Hamard, F. Pereira et al., "Image quality and dose reduction opportunity of deep learning image reconstruction algorithm for CT: a phantom study," European Radiology, vol. 30, no. 7, pp. 3951-3959, 2020.

[10] D. Ippolito, L. Riva, C. R. Talei Franzesi et al., "Diagnostic efficacy of model-based iterative reconstruction algorithm in an assessment of coronary artery in comparison with standard hybrid-Iterative reconstruction algorithm: dose reduction and image quality," La radiologia medica, vol. 124, no. 5, pp. 350-359, 2019.

[11] G. Wang and J. K. McKenney, "Urinary bladder pathology: world health organization classification and American joint committee on cancer staging update," Archives of Pathology \& Laboratory Medicine, vol. 143, no. 5, pp. 571-577, 2019.

[12] S. K. Khiste, S. B. Hosain, Y. Dong et al., "Incorporation of fluorescence ceramide-based HPLC assay for rapidly and 
efficiently assessing glucosylceramide synthase in vivo," Scientific Reports, vol. 7, no. 1, p. 2976, 2017.

[13] W. Stiller, "Basics of iterative reconstruction methods in computed tomography: a vendor-independent overview," European Journal of Radiology, vol. 109, pp. 147-154, 2018.

[14] Y. Kubo, K. Ito, M. Sone et al., "Diagnostic value of modelbased iterative reconstruction combined with a metal artifact reduction algorithm during CT of the oral cavity," American Journal of Neuroradiology, vol. 41, no. 11, pp. 2132-2138, 2020.

[15] S. Lombardi, L. Riva, M. Patassini et al., "“Hyperdense artery sign" in early ischemic stroke: diagnostic value of modelbased reconstruction approach in comparison with standard hybrid iterative reconstruction algorithm," Neuroradiology, vol. 60, no. 12, pp. 1273-1280, 2018.

[16] S. Ichikawa, M. Hamada, D. Watanabe, O. Ito, T. Moriya, and H. Yamamoto, "Optimal slice thickness of brain computed tomography using a hybrid iterative reconstruction algorithm for identifying hyperdense middle cerebral artery sign of acute ischemic stroke," Emergency Radiology, vol. 28, no. 2, pp. 309-315, 2021.

[17] S. J. Gardner, W. Mao, C. Liu et al., "Improvements in cbct image quality using a novel iterative reconstruction algorithm: a clinical evaluation," Advances in Radiation Oncology, vol. 4, no. 2, pp. 390-400, 2019.

[18] C. Miller, D. Mittelstaedt, N. Black et al., "Impact of CT reconstruction algorithm on auto-segmentation performance," Journal of Applied Clinical Medical Physics, vol. 20, no. 9, pp. 95-103, 2019.

[19] H. Kawashima, K. Ichikawa, K. Matsubara, H. Nagata, T. Takata, and S. Kobayashi, "Quality evaluation of imagebased iterative reconstruction for CT: comparison with hybrid iterative reconstruction," Journal of Applied Clinical Medical Physics, vol. 20, no. 6, pp. 199-205, 2019.

[20] A. Löve, R. Siemund, P. Höglund et al., "Hybrid iterative reconstruction algorithm in brain CT: a radiation dose reduction and image quality assessment study," Acta Radiologica, vol. 55, no. 2, pp. 208-217, 2014.

[21] L. Lambert, M. Novak, M. Siklova, E. Krauzova, V. Stich, and A. Burgetova, "Hybrid and model-based iterative reconstruction influences the $\mathrm{v}$ of visceral and subcutaneous adipose tissue on ultra-ldose CT," Obesity, vol. 28, no. 11, pp. 2083-2089, 2020.

[22] S. K. Kim, C. Kim, K. Y. Lee et al., "Accuracy of model-based iterative reconstruction for CT volumetry of part-solid nodules and solid nodules in comparison with filtered back projection and hybrid iterative reconstruction at various dose settings: an anthropomorphic chest phantom study," Korean Journal of Radiology, vol. 20, no. 7, pp. 1195-1206, 2019.

[23] C. De Margerie-Mellon, C. De Bazelaire, C. Montlahuc et al., "Reducing radiation dose at chest CT," Academic Radiology, vol. 23, no. 10, pp. 1246-1254, 2016. 